\title{
57 cohortes respaldan el valor pronóstico del índice de masa corporal
}

\author{
57 cohorts studies support the pronostic value of body mass index
}

Prospective Studies Collaboration. Lancet 2009;373:1083-96.

\section{Objetivo}

Definir el rol del índice de masa corporal (IMC) como predictor de mortalidad, analizando su asociación con indicadores de mortalidad general y por causas específicas.

\section{Diseño, lugar y pacientes}

Estudio analítico secundario de datos de 57 estudios prospectivos observacionales mayoritariamente europeos y de América del Norte (seguimiento de 894.576 adultos sanos). Criterios de exclusión: IMC igual o mayor a $50 \mathrm{~kg} / \mathrm{m} 2$ ó menor a $15 \mathrm{~kg} / \mathrm{m} 2$, antecedentes de enfermedad cardiovascular, falta de seguimiento entre los 35 y los 89 años.

\section{Evaluación de los factores pronósticos y medición de los} resultados principales

Desde el inicio del seguimiento se recogió información demográfica, IMC, tensión arterial, colesterol HDL y LDL, consumo de tabaco y alcohol y diabetes mellitus; registrándose además las causas de muerte. El análisis excluyó los casos en quienes la muerte se presentó durante los primeros cinco años del seguimiento con el fin de atenuar el posible efecto confundidor $^{*}$ que pudieran tener enfermedades pre-exsitentes que simultáneamente aumentan la mortalidad y el IMC.

El análisis identificó patrones de mortalidad general y por causas específicas por estratos de IMC a través del cálculo de los cocientes de riesgos instantáneos (del inglés: "hazard ratio", HR) ajustando por estudio, sexo, edad y tabaquismo, pero no por tensión arterial, lípemia o diabetes, ya que son mecanismos a través de los cuales el IMC afecta la mortalidad cardiovascular.

\section{Resultados principales}

En ambos sexos la mortalidad más baja se dio entre los participantes con IMC de 22,5 a $25 \mathrm{~kg} / \mathrm{m} 2$. Por encima de este rango fueron registradas asociaciones positivas que se resumen en la tabla 1.
Tabla 1: principales resultados.

\begin{tabular}{l|l|c}
\multicolumn{2}{c|}{ Indice de masa corporal (kg/m2) } & Hazard ratio (IC95\%) \\
\hline \multirow{2}{*}{ Valor de referencia } & $\mathbf{2 2 , 5}$ a $\mathbf{2 5} \mathbf{~ k g} / \mathbf{m} 2)$ & 1 \\
\hline \multirow{2}{*}{$\begin{array}{c}\text { Aumento por cada } \\
\text { kg } / \mathrm{m}^{2} \text { de aumento }\end{array}$} & Mortalidad general & $1,29(1,27$ a 1,32$)$ \\
\cline { 2 - 3 } del indice de masa & Mortalidad cardiovascular & $1,41(1,37$ a 1,45$)$ \\
\cline { 2 - 3 } corporal & Mortalidad por causa renal & $1,59(1,27$ a 1,99$)$ \\
\cline { 2 - 3 } & Mortalidad de causa hepática & $1,82(1,59$ a 2,09$)$ \\
\cline { 2 - 3 } & Mortalidad respiratoria & $1,10(1,06$ a 1,15$)$ \\
\cline { 2 - 3 } & Incidencia de diabetes & $2,16(1,89$ a 2,46$)$ \\
\hline
\end{tabular}

Por debajo del rango establecido, el IMC se asoció inversamente con la mortalidad general (HR por cada $5 \mathrm{~kg} / \mathrm{m}^{2}$ más de IMC=0,79; IC95\% 0,77 a 0,82). Esta asociación inversa fue especialmente fuerte para la mortalidad por enfermedades respiratorias, cáncer de pulmón y cáncer del tracto digestivo alto (tanto en fumadores como en no fumadores) y fue mucho más marcada en el primer grupo.

\section{Conclusiones}

EI IMC es un fuerte predictor de mortalidad general, tanto por encima como por debajo de su aparente valor óptimo de 22,5 a $25 \mathrm{~kg} / \mathrm{m}^{2}$. El aumento progresivo de mortalidad por encima de este rango es debido principalmente a enfermedades cardiovasculares, con mecanismos causales muy consensuados. Por debajo del valor óptimo, el aumento del riesgo está dado por enfermedades relacionadas con el tabaquismo, sin estar del todo dilucidados los mecanismos causales.

Palabras claves: índice de masa corporal, mortalidad, factores de riesgo, estudio prospectivo, estudio observacional.

Key words: body masss index, mortality, risk factors, prospective study, observacional study. Fuente de financiamiento: UK Medical Research Council, British Heart Foundation, Cancer Research UK, EU BIOMED programme, US National Institute on Aging, Clinical Trial Service Unit (Oxford, UK).

\section{Comentario}

El IMC es una razonable medida de adiposidad general. Índices elevados constituyen hoy factores de riesgo para enfermedades prevalentes como la patología cardiovascular, aunque existen aspectos aún no dilucidados como la disminución paradojal de la mortalidad reportada en pacientes con insuficiencia cardíaca e IMC elevados ${ }^{1,2,3}$.

Además del IMC, en la práctica clínica usualmente disponemos de otras medidas y estudios para definir el perfil pronóstico y los factores de riesgo de nuestros pacientes. Este trabajo de gran tamaño muestral expone la gran capacidad predictiva del IMC como único elemento, sin tener en cuenta otras referencias clínicas. Es por ello que para los ajustes se han tenido en cuenta factores que condicionan por separado el IMC y la mor- talidad, como el tabaquismo, pero no aquellas variables que se sitúan en el camino de la causalidad a través del cual el IMC determina la mortalidad, como son la tensión arterial, el nivel de lípidos y la diabetes.

\section{Conclusión de la comentadora}

Sería recomendable tener en cuenta los resultados de este estudio a la hora de enfatizar los hábitos de vida saludables. Por ejemplo, evitando un incremento de su IMC de 24 a $32 \mathrm{~kg} / \mathrm{m}^{2}$ un adulto joven ganaría en promedio tres años mas de vida.

Julieta Galante [ Instituto de Efectividad Clínica y Sanitaria ] jgalante@iecs.org.ar

Recibido el 22/05/09 y aceptado el 02/06/09

Galante J. 57 cohortes respaldan el valor pronóstico del índice de masa corporal. Evid Act Pract Ambul. 12(3).96. Jul-Sep.2009. Comentado de: Prospective Studies Collaboration. Lancet 2009;373:1083-96. PMID:

Disponible en URL: http://www.thelancet.com/journals/lancet/article/PIIS0140-6736(09)60318-4/fulltext (último acceso 26/05/09).

Referencia

1. Oreopoulos A y col. Body mass index and mortality in heart failure: a meta-analysis. American heart journal, 2008. 156(1): p. 13-22.

2. Manson J y col. A prospective study of obesity and risk of coronary heart disease in women. The New England journal of medicine, 1990.322(13): p. 882-9.

3. Song Y y col. Body mass index and ischemic and hemorrhagic stroke: a prospective study in Korean men. Stroke; a journal of cerebral circulation, 2004. 35(4):p.831-6. 